For liquid hydrogen iodide we find a Raman line of a very diffuse character. As in the case ${ }^{2}$ of hydrogen chloride and bromide, it has a complex structure. The wave numbers in the liquid and solid state as compared with the gaseousstatearegiven in the accompanying table. The structure of the line in the liquid may be seen from Fig. 1. The points marked on the curve have the following wave numbers : $a, 2178 \mathrm{~cm}^{-1}$; $b, 2167 \mathrm{~cm}^{-1} ; c, 2165 \mathrm{~cm}^{-1} ; d, 2162.5 \mathrm{~cm}^{-1} ; e$, $2151 \mathrm{~cm} .^{-1}$. It may be emphasised that the structure and the wave numbers are not very accurately known.

From these results, it will be seen that although the low temperature apparatus already used is quite adequate for our purpose, a spectrograph of higher dispersion will be necessary if we are to carry this investigation further. We have therefore begun to construct a new type of spectrograph with a liquid prism.

Physikal. Chem. Institut d. Universität,

H. EPSTEIN.

W. STEINER.

Berlin.

Laboratory of Physical Chemistry, Cambridge.

May 2.

1 Grassmann, Z. Phys., 82, 767 ; 1933.

E O. Salant and A. Sandow, Phys. Rev., 37, 373; 1931. E. 0. Salant and D. Callihan, Phys. Rev., 43, $590 ; 1933$.

\section{Magnetic Moment of the Deuton}

IN a previous note ${ }^{1}$ we reported, together with Mr. Frisch, on experiments concerning the deflection of a beam of 'ordinary' hydrogen molecules in an inhomogeneous magnetic field. From these experiments, we were able to derive the magnetic moment of the proton. The value obtained was 2.5 nuclear magnetons (not 1, as expected theoretically).

We have now performed similar experiments with a beam of 'heavy' hydrogen molecules and derived in a similar way the magnetic moment of the deuton. The value obtained is about 0.7 nuclear magnetons ${ }^{2}$.

A detailed account of these experiments will appear in the Physical Review.

\section{EstermanN. O. STERN.}

Carnegie Institute of Technology, Pittsburgh, Pa. May 10.

1 NatURe, 132, 169, July 29, 1933.

The value given in the Bulletin of the American Physical Society (vol. 9, p. 29, 1934, No. 2) is wrong, due to an error in the calculations.

\section{Electron Microscopy of Biological Objects}

Is a recent paper Ruska ${ }^{1}$ demonstrated experimentally the possibility of surpassing considerably the resolving power of an ordinary microscope by the use of an electron microscope. This high resolving power cannot be applied in biological research, how. ever, without developing a new histological technique to prevent the destruction of the organic cells by the intense electronic bombardment.

To overcome this difficulty, it seems that there are the following possibilities :

(1) Intense cooling of the object (for example, by contact with an extremely thin metal foil which is cooled by conduction).

(2) Impregnating the object with a substance which makes the object less destructible.

(3) Impregnating the object in such a way that a framework of the object is preserved although the object itself is destroyed.

(4) Combining methods (1) and (2), or (1) and (3).

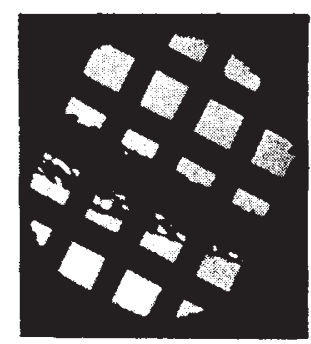

FIG. $1 . \times 65$.

We obtained the best results by using the third method. To arrive at good results by this method the following conditions must be satisfied by the metallic or other framework: It must be $(a)$ geometrically similar to the object; $(b)$ of high melting point and good thermal conductivity; and $(c)$ of high atomic weight.

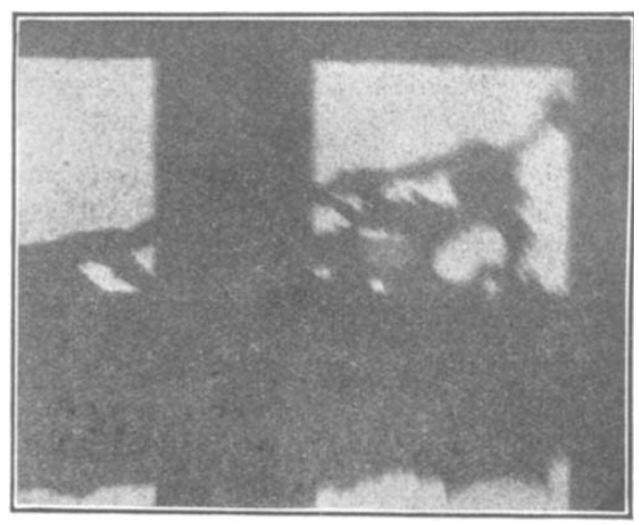

FIG. 2. $\times$ about 450 .

Figs. I and 2 show the results we obtained. They represent a $15 \mu$ section of a Drosera intermedia leaf on a copper net. Fig. 1 is enlarged 65 times and Fig. 2 about 450 times. The resolving power can be estimated from the sharpness of the border of the copper wire; it corresponds to about $1 \mu$. The microscopic object was impregnated with osmium, as is done in the usual microscopy. From the above photographs, it seems that the osmium impregnation method can be applied-perhaps with some modifications-to electron microscopy.

The photomicrographs were taken with an electronmicroscope of the magnetic type, the description of which will be published later.

University of Brussels.

L. Marton. May 7.

${ }^{1}$ E. Ruska, Z. Phys., 87, 580 ; 1934. 